\title{
Bilinguals' Vocabulary Preferences in Advertising: The Difference Between English and Chinese Copy—An Abstract
}

\author{
Chun Zhang, Michel Laroche, and Marie-Odile Richard
}

\begin{abstract}
Given the language as an important cultural factor in studying consumer's purchase behavior, the current study explores the vocabulary use and preference by bilinguals (Semin 2012). Different vocabulary words are tested in both English and Chinese contexts. Two studies demonstrate consumers' different preferences for adjectives, verbs, and nouns. Through self-reported questionnaires and scale-measured surveys, the current study shows that in general the use of verbs is more salient in the Chinese contexts, while the use of adjectives and nouns is more prominent in the English contexts. We also obtain an interaction of language and vocabulary for purchase intentions. In the purchase decision process, when bilingual consumers are exposed to the Chinese copy, nouns play an essential role in affecting their purchase intentions. When exposed to the English copy, adjectives play a more effective role. Taken together, the current research provides a strong evidence of word type effect for bilinguals. The results extend the previous vocabulary study into non-interpersonal contexts, and focus on bilinguals. In addition, the current research contributes to theoretical development as well as managerial implications.
\end{abstract}

References available upon request.

C. Zhang $(\varangle) \cdot$ M. Laroche

Concordia University, Montréal, QC, Canada

e-mail: chun.zhang@concordia.ca; michel.laroche@concordia.ca

M.-O. Richard

SUNY Polytechnic Institute, Utica, NY, USA

e-mail: MarieOdile.Richard@sunyit.edu 